\title{
Éditorial \\ Le développement territorial en Outaouais : spécificités des trajectoires et de la recherche
}

\author{
Guy Chiasson, professeur-chercheur \\ Université du Québec en Outaouais
}

Chantale Doucet, professionnelle de recherche

Centre de recherche en développement territorial

L'Outaouais a longtemps été considérée comme une région avec une réalité distincte de l'ensemble québécois. Le constat souvent fait par les acteurs régionaux voulant que l'Outaouais ait été négligée historiquement par le gouvernement du Québec n'a pas perdu de son actualité comme en témoignent les revendications bien présentes dans les discours politiques pour un statut particulier en éducation et en santé notamment. La différence régionale outaouaise associée à sa proximité avec l'Ontario a également été mise en relief dans les recherches universitaires. Par exemple, L'histoire de l'Outaouais (Gaffield, 1994), une des grandes synthèses de l'histoire régionale, comparait l'Outaouais aux « deux faces de Janus », à la fois tournées vers le Québec et vers l'Ontario. Bien qu'elle ait joué un rôle constant dans le développement de la région, la proximité avec l'Ontario et Ottawa, la capitale nationale a pris un élan considérable à partir des années 1970. Comme l'a bien démontré André Beaucage (1994) dans son chapitre sur l'Histoire de l'Outaouais, les années 1970 ont été marquées par l'effondrement $\mathrm{du}$ secteur manufacturier forestier et par l'accentuation de la tertiarisation de l'économie régionale avec la présence accrue des bureaux de la fonction publique fédérale sur la portion québécoise de la région de la capitale nationale.

Des analyses plus sensibles aux disparités géographiques (Doucet, Favreau et Robitaille, 2008) ont par la suite montré que ces phénomènes de tertiarisation de l'économie régionale n'ont pas la même portée pour toutes les MRC de la région. L'actuelle Ville de Gatineau et sa frange périurbaine (en gros la MRC des Collines-del'Outaouais) sont assez fortement intégrées à l'espace métropolitain transfrontalier partagé avec
Ottawa. L'omniprésence des bureaux du gouvernement fédéral dans cet espace métropolitain a contribué au dynamisme du marché du travail et à une croissance démographique soutenue dans le secteur urbain et périurbain. Une analyse récente a toutefois révélé que ce dynamisme a nettement reculé depuis 2011 avec les compressions d'emplois dans la fonction publique fédérale (Doucet, 2014).

Pour leur part, les territoires plus éloignés du centre (grossièrement, les MRC Pontiac, Papineau et Vallée-de-la-Gatineau) ont une économie beaucoup moins dynamique parce qu'encore dépendante en bonne partie du secteur forestier en déclin (Chiasson, 2005; Chiasson et al., 2010). C'est donc dire que des réalités territoriales fort différenciées cohabitent dans l'Outaouais contemporain: des territoires métropolitains qui bénéficient au plan de l'emploi de leur proximité avec Ottawa et des territoires ruraux en marge des dynamiques métropolitaines avec des trajectoires de développement qui rappellent d'assez près celles des régions ressources québécoises. Ces tendances contrastées contribuent largement à différencier l'Outaouais de la plupart des autres régions québécoises.

L'intérêt des chercheurs pour le développement territorial en Outaouais est assez récent. Jusqu'aux années 2000, l'Université du Québec en Outaouais, seule université en région, n'avait pas de programme de formation, ni de programme de recherche consacré au développement régional. De façon assez révélatrice, la grande synthèse de l'histoire régionale avait été réalisée principalement par des chercheurs de l'Université d'Ottawa (Gaffield et al., 1994). Cependant, depuis les années 2000, l'Outaouais s'est doté d'une capacité 
de recherche distincte. La question du développement des régions et en particulier celle de l'Outaouais occupe, en effet, une place grandissante dans l'enseignement et la recherche à l'UQO ce qui a permis d'éclairer de nombreuses facettes $\mathrm{du}$ développement territorial et de favoriser une meilleure connaissance du profil territorial singulier de la région.

L'objectif de ce numéro d'Organisations et territoires est d'offrir un aperçu des travaux de recherches réalisés sur le développement territorial de l'Outaouais. Ces travaux, réunis en neuf articles, dévoilent diverses stratégies et pratiques pour animer le développement et l'innovation sur le territoire tout en apportant une réflexion critique sur les dynamiques et pratiques territoriales. Les auteurs sont principalement des chercheurs et des étudiants diplômés de l'UQO mais nous avons également fait appel à des partenaires et acteurs pour qu'ils partagent leur connaissance fine des trajectoires de développement territorial. Collectivement, ces textes permettent de documenter une réalité régionale qui reste assez méconnue et d'en dégager les spécificités propres. Néanmoins, nous croyons que la variété des situations et des approches qui caractérise la réalité de l'Outaouais intéressera non seulement les acteurs et chercheurs d'ici, mais également ceux d'autres territoires. Le caractère pluridimensionnel du développement territorial est en effet mis de l'avant avec un contenu à saveur économique, sociale et démographique qui prend en compte les réalités urbaines et rurales, rejoignant du même coup l'intérêt d'un plus large lectorat.

Par ailleurs, ce numéro a été écrit à l'hiver et au printemps 2015 dans un contexte de changements majeurs dans la gouvernance des régions marquée par les réformes du gouvernement québécois en matière de développement territorial. Nous ne pouvions passer sous silence ce contexte qui teinte le contenu de plusieurs textes et modifie les perspectives d'avenir en Outaouais et ailleurs au Québec. Quels sont ces principaux changements et quels enjeux cachent-ils? Sous la plume de JeanFrançois Simard et d'Yvon Leclerc, le premier article vient contextualiser ces changements en cours dans le modèle québécois de développement territorial. Les chercheurs font ainsi un retour sur les raisons qui ont motivé la mise en place de ce modèle qui misait à l'origine sur l'intégration de la société civile comme partenaire du développement. Ils questionnent, non sans une inquiétude certaine, les logiques sur lesquelles s'appuient les transformations récentes de la politique régionale québécoise.

Les deux articles qui suivent posent un regard sur les pratiques de planification et d'aide à la décision mobilisées par les acteurs du milieu pour stimuler le développement. L'article de Martin Robitaille, Élodie Plassin et Amandine Cochard présente une démarche audacieuse initiée par les acteurs de l'Outaouais qui ont choisi d'expérimenter la méthode d'analyse prospective territoriale afin de doter la région d'une vision socioéconomique. L'équipe de chercheurs qui a accompagné les acteurs dans cette démarche expose les principales étapes, la valeur ajoutée et les défis de cette pratique encore méconnue au Québec.

L'article de Normand Bourgault et ses coauteurs examine les nombreux documents de planification de projets récréotouristiques réalisés en Outaouais rural au cours des dernières années. Cet examen, qui s'appuie sur une approche rigoureuse de gestion de projet, révèle plusieurs lacunes qui freinent l'ensemble du processus menant à la réalisation de ces projets. Sur cette base, les auteurs proposent un modèle théorique de faisabilité des projets qui contribue à améliorer l'efficacité de ce processus.

Pour accompagner les exercices de planification et de prospective, des mécanismes participatifs sont souvent mobilisés afin d'intégrer les savoirs des populations et des acteurs du milieu. La participation du public est d'ailleurs au cœur du renouvellement de diverses pratiques d'aménagement et de développement à l'échelle locale et régionale au Québec. Dans ce contexte, l'article de Lynda Gagnon et Mario Gauthier scrute la démarche originale de planification collaborative expérimentée par la Ville de Gatineau, quatrième ville d'importance au Québec, qui a choisi d'impliquer le public à toutes les étapes du processus de révision de son schéma d'aménagement et de développement. À travers l'examen de cette démarche, les auteurs évaluent l'influence réelle du public dans la prise de décision et les enjeux qui affectent la démarche.

Dans la même veine, divers mécanismes de concertation ont été mis en place au Québec. Issues 
de la décentralisation de la gestion forestière, les Commissions régionales sur les ressources naturelles et le territoire implantées dans les régions du Québec en 2006 sont l'un de ces mécanismes. L'article d'Édith Leclerc s'intéresse aux apprentissages et aux obstacles liés à la mise en place de cette commission en Outaouais ainsi qu'à son rôle dans la planification du développement des ressources naturelles sur le territoire avec l'élaboration et la mise en œuvre de plans régionaux.

De moindre envergure, les enjeux de l'immigration et de la jeunesse ont également mené à une stratégie de partenariat et de participation alliant une diversité d'acteurs en Outaouais. S'appuyant sur les réflexions de ces acteurs, l'article de Chantale Doucet présente cette démarche participative en insistant sur les défis rencontrés pour assurer une durabilité des ressources et des services existants tout en prenant en compte les besoins et spécificités des différents territoires de l'Outaouais.

Les trois derniers articles de la revue s'intéressent à l'entrepreneuriat à partir de trois principales entrées. Le premier article donne la parole à deux commissaires en développement d'entreprises, Simon Ouellet et Renald A. Piché, qui expliquent l'évolution et les rouages du fonctionnement et des pratiques de Développement économique - CLD Gatineau pour accompagner les entrepreneurs. L'article répond certes à une lacune, car encore trop peu de recherches ont contribué à mieux comprendre le rôle et les pratiques des organisations de développement local au Québec. Malgré la vague de compression qui a mené à l'abolition de la majorité des CLD au Québec, les acteurs gatinois ont choisi de préserver cette organisation qui a développé au fil des années un modèle unique adapté aux contextes et spécificités de l'entrepreneuriat local.

L'Outaouais se démarque également par une croissance et un dynamisme élevé de l'entrepreneuriat collectif. Guy Chiasson, Patrick Duguay et Claudine Lalonde dressent un portrait de l'importance de l'économie sociale dans le développement de l'Outaouais pour ensuite s'attarder à deux études de cas : la création de la Laiterie de l'Outaouais dans un domaine en perte de vitesse au Québec et la Coopérative Place du Marché dans une municipalité dévitalisée. La mobilisation citoyenne et l'identité régionale sont parmi les principales ressources territoriales qui ont contribué à la concrétisation de ces projets.

On observe également en Outaouais une nouvelle vague entrepreneuriale liée à l'agriculture et l'agroalimentaire. Le dernier article de Chantale Doucet s'intéresse à l'émergence de ce nouveau «créneau » influencée par le contexte transfrontalier de l'Outaouais qui procure aux entrepreneurs des avantages certains, mais tout autant de défis. Les critères qui caractérisent les spécificités de ce créneau et son potentiel sont décrits pour ensuite poser un regard sur les stratégies d'autres régions transfrontalières afin d'alimenter les pistes de réflexion et d'action pour l'Outaouais. L'article contribue également à éclairer la compréhension de la spécificité transfrontalière qui caractérise la région.

\section{BIBLIOGRAPHIE}

Beaucage André (1994). De la manufacture aux services, dans Histoire de l'Outaouais, sous la dir. de Chad Gaffield et al., Institut québécois de recherche sur la culture, Québec.

Chiasson, Guy, Édith Leclerc et Caroline Andrew (2010). La multifonctionnalité forestière à l'épreuve de la distance : réflexion à partir de deux cas forestiers. $L a$ multifonctionnalité de l'agriculture et des territoires ruraux: enjeux théoriques et d'action publique, Rimouski : GRIDEQ.

Chiasson, Guy (2005). L'attractivité des territoires dans un contexte de mondialisation: Quel espoir pour les milieux ruraux? Les nouveaux facteurs d'attractivité dans le jeu de la mondialisation, Rennes: Presses universitaires de Rennes, 2005. 131-142.

Doucet, Chantale (2014). Portrait économique de l'Outaouais 2014. Rapport réalisé pour l'UQO et le Comité de développement économique régional de l'Outaouais de la Chambre de commerce de Gatineau, $53 \mathrm{p}$.

Doucet, Chantale, Louis Favreau et Martin Robitaille (dir.) (2007). L'Outaouais une région qui gagne et qui perd. Enjeux démographiques et économiques. Publication de la Chaire de recherche du Canada en développement des collectivités et Centre de recherche en développement territorial, Université du Québec en Outaouais, $324 \mathrm{p}$.

Gaffield, Chad et al. (1994). Histoire de l'Outaouais. Québec, IQRC, 876 p. 
2015

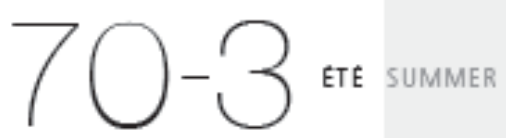

REVUE TRIMESTRIELLE

RELATIONS INDUSTRIELLES

Revue trimestrielle bilingue publiée depuls 1945 par le Département des relations Industrielles de I'Université Laval

EDITORIAL

Les 70 ans de RI/R RUR celebrates its $70^{\text {th }}$ year PAR MARTINE D'AMOURS

ARTICLES

Advancing Industrial Relations Theory: An Analytical Synthesis of British-American and Pluralist-Radical Ideas BRUCE E. KAUAMAN AND GREGOR GALL

Taxonomie des conflits entre le travail et la famille: une analyse multidimensionnelle

à l'aide de cartes auto-organisatrices THIERRY WIS ET AZZZ RHNMA

The Economic Impact of the Great Recession on Aboriginal People Living off Reserve in Canada DANIELEE LAMB

Candidatures spontanées, réseaux et intermédiaires publics: quelle information et quels appariements sur le marché du travail français? GUILLEMETTE DE LARQUIER ET GERALDINE RUEUCAU

Adjustments to Minimum Wages in China: Cost-Neutral Offsets JING WANG AND MORLEY GUNDERSON

Les politiques de formation professionnelle dans les services à domicile.

Influence de la structure de marché et du dialogue social en France et en Belgique ETIENNE COGNARD

The Prospects for Greater Enforcement of Teen Employment Laws in Alberta, Canada:

"Politically, how do you make it relevant? [...] Kill more young people! " BOB BARNETSON

\section{INDUSTRIAL RELATIONS}

\section{QUARTERLY REVIEW}

A bilingual quarterly published since 1945 by the Industrlal Relations Department, Université Laval

\section{RI/IR EN LIGNE}

RUR est disponible en ligne sur le site Erudit :

www.erudit.org/revue/ri

Pour un abonnement institutionnel, contacter Erudit.

Pour consulter les règles de publication ou pour vous abonner, visitez notre site Internet : www.riir.ulaval.ca

\section{RI/IR ONLINE}

$R$ R is available on line on Erudit website at: www.erudit.org/revue/ri

For an institutional subscription to digitalized issues, please contact Erudit.

Visit our website for Notes to contributors or to subscribe: www.riir.ulaval.ca

\section{RELATIONS INDUSTRIELLES} INDUSTRLAL RELATIONS

Pavillon J.-A.-DeSève 1025 , avenue des Sclences-Humalnes Bureau 3129, Unwersite Laval Québec (Québec) Canada G1V OA6

TELEPHONE : (418) 656-2468

COURRIEL/E-MAIL :

relat.Ind@rit.ulaval.ca

www.rllr.ulaval.ca 